SALAZAR, C. -1996- Estudio Fitosociológico de la Vegetación riparia andaluza (provincia Bética): Cuenca del Guadiana Menor. Tesis Doctoral. Universidad de Jaén.

SALAZAR, C., E. CANO y F. VALLE -1996Aportaciones a la flora vascular de las provincias de Granada y Jaén (S. España). Acta Bot. Malacitana 21: 314-318.

SALAZAR, C., E. ARROJO, J.A. TORRES, A. GARCÍA-FUENTES y F. VALLE -2000Algunos elementos de interés en la flora edafófila de la provincia de Granada (S. España). Acta Bot. Malacitana 25:240-243.

SALAZAR, C., A. GARCÍA-FUENTES y F. VALLE -2001- Flora vascular y fitocenosis endémicas, raras y amenazadas en los ríos y humedales de la cuenca del Guadiana Menor (Sureste de España): Áreas y localidades de interés. Bol. Real Soc. Esp. Hist. Nat. (Sec. Biol.) 96 (3-4): 99-116.

SORIANO, C. y C. CEBOLLA - 1981- Contribución al conocimiento de la flora de Segura-Cazorla (Andalucía, España). Lazaroa 3: 219-225.

VOGT, R. -1993- Cochlearia L. In: Castroviejo, S., C. Aedo, C. Gómez Campo, M. Laínz, P.
Montserrat, R. Morales, F. Muñoz Garmendia, G. Nieto Feliner, E. Rico, S. Talavera \& L. Villar (eds.). Flora iberica 4: 227-233. Real Jardín Botánico de Madrid. CSIC. Madrid.

VV.AA. -2000- Lista Roja de Flora Vascular Española (valoración según categorías UICN).

Conservación Vegetal 6 (extra): 11-38.

WILLKOMM, M. \& J. LANGE -1861/1880-

Prodromus Florae Hispanicae. 3 Tomos. Stuttgart.

Aceptado para su publicación en marzo de 2002

Dirección de los autores. C. SALAZAR y J.A. TORRES: Departamento de Biología Animal, Biología Vegetal y Ecología, Facultad de Ciencias Experimentales, Universidad de Jaén, Campus Las Lagunillas. E-23071. Jaén. España. E-mail: csalazar@ujaen.es; J.A. ALGARRA y E. CAÑADAS: Departamento de Biología Vegetal, Facultad de Ciencias, Universidad de Granada, Campus Fuentenueva. E-18001. Granada. España.

\title{
82. AMPLIACIÓN AL CONOCIMIENTO DE LOS MACRÓFITOS ACUÁTICOS DE LA PROVINCIA DE MÁLAGA
}

\author{
Fernando ORTEGA, Gema PARRA y Francisco GUERRERO
}

Contributions to the knowledge of aquatic macrophytes in Málaga province

Palabras clave. Macrófitos acuáticos, corología, humedales, Málaga

Keywords. Aquatic macrophytes, chlorology, wetlands, Málaga

Una gran cantidad de trabajos han puesto de manifiesto la importancia de los macrófitos en el funcionamiento de los ecosistemas acuáticos en general y de los humedales en particular (e.g. Landers, 1982; Downing y Anderson, 1985; Blindow, 2000). Recientemente 
la presencia de estos organismos ha sido igualmente utilizada como bioindicadores en la valoración de los humedales, tanto en nuestro país (Cirujano et al., 1992) como fuera del mismo (Goslee et al., 1997). Sin embargo, la información existente no es completa y el estudio necesario para la correcta valoración de nuestros humedales debe basarse en unos datos más detallados sobre la vegetación acuática presente en los mismos, que precisa por tanto de nuevas herborizaciones, así como de estudios específicos (Cirujano, 1995).

Por otro lado, numerosas zonas húmedas mediterráneas son hábitats temporales caracterizados por una secuencia de inundación y desecación, de gran variabilidad interanual, que condiciona los ciclos biológicos de los macrófitos acuáticos en ellas presentes (Grillas et al., 1993). Este hecho origina que la vegetación acuática presente igualmente, distintos periodos de presencia-ausencia en consonancia con los periodos de inundación y por ende con las características físico-químicas de las aguas. Además de estos ritmos de inundación y desecación, los procesos de eutrofización, colmatación, etcétera, condicionan igualmente cambios en la composición de la vegetación acuática que deben ser evaluados a través de estudios interanuales. En esta línea, el presente trabajo pretende aportar datos destinados a la recogida de esta información, fundamental para una futura gestión de nuestros humedales, bien a través del estudio de la vegetación acuática en nuevos humedales, como en humedales previamente muestreados.

La zona objeto de estudio, la comarca de Antequera, se sitúa dentro del núcleo endorreico más meridional de la Península Ibérica, el denominado endorreismo andaluz o endorreismo Bético (Dantín, 1940). Esta comarca se sitúa al norte de la provincia de Málaga, perteneciendo desde un punto de vista corológico al sector Subbético de la provincia Bética. Geológicamente son las formaciones del Keuper (Trias) las que ofrecen un mayor interés por su incidencia en la composición salina de los humedales, y por tanto en su vegetación (Martínez Parras, 1984). Para una mayor información sobre la vegetación acuática presente en esta comarca y en el conjunto de humedales de la provincia de Málaga, se pueden consultar, entre otras, las referencias citadas en Nieto et al. (1997).

Los humedales estudiados han sido la laguna de la Serafina Chica (Humilladero), laguna formada en una cantera del cerro del Palo (Fuente de Piedra), laguna de la Ratosa (Alameda), laguna de Siete Novias (Antequera), laguna del Viso (Antequera), laguna de la Caja (Antequera), laguna de las Lomas (Antequera), laguna-charca ganadera junto al cortijo de Aguilar (Antequera), laguna Grande (Archidona), laguna Chica (Archidona), manantial y un par charcas artificiales (fundamentalmente para la actividad ganadera) en la zona de los Hoyos (Archidona). El material recolectado se encuentra depositado en el Herbario Jaén (Facultad de Ciencias Experimentales, Universidad de Jaén). Los manuales o publicaciones utilizados para la identificación del material recolectado han sido Comelles (1985); Talavera et al. (1986); García Murillo y Talavera(1986); Valdés et al. (1987) y Velayos (1988).

Señalar que junto a la información obtenida se hace referencia, en función de la bibliografía consultada, a aquellas citas nuevas para la provincia de Málaga:

\section{Characeae}

\section{Chara vulgaris $\mathrm{L}$.}

Forma praderas sumergidas, propia de aguas corrientes o estancadas, de dulces a hiposalinas.

MÁLAGA. Archidona: Manantial, 30SUG8307; F. Ortega, 25-07-01 (J-61-474); Laguna Grande, 30SUG8407; F. Ortega, 25-07-01 (J-61-475).

\section{Chara hispida $\mathrm{L}$.}

Relacionada con sistemas de aguas permanentes o temporales, de una amplia gama de salinidades (dulces a mesohalinas). Primera cita provincial.

MÁLAGA. Humilladero: Laguna de la Serafina Chica, 30SUG4716; F. Ortega, 19-06-01 (J-61-476). 
Chara hispida var. major (Hartm.) R.D. Wood

En aguas permanentes y generalmente profundas. Primera cita provincial.

MÁLAGA. Archidona: Laguna Grande, 30SUG8407; F. Ortega, 25-07-01 (J-61-477).

\section{Chara connivens Salzm. ex A. Braun.}

Forma parte de comunidades de carófitos que colonizan aguas dulces a hiposalinas y generalmente estacionales, así como charcas ganaderas y balsas de riego. Primera cita provincial.

MÁlAGA. Antequera: Laguna de Siete Novias, 30SUG6418; F. Ortega, 19-06-01 (J-61478); Laguna del Viso, 30SUF6997; F. Ortega, $10-$ 07-01 (J-61-479); Laguna de la Caja, 30SUF6998; F. Ortega, 10-07-01 (J-61-480); Cortijo de Aguilar, 30SUF6899; F. Ortega, 10-07-01 (J-61-481); Laguna de las Lomas, 30SUF7198; F. Ortega, 10-07-01 (J61-482). Archidona: Charca Los Hoyos, 30SUG8505; F. Ortega, 10-07-01 (J-61-483); Charca ganadera, 30SUG7400; F. Ortega, 25-07-01 (J-61-484); Laguna Grande, 30SUG8407; F. Ortega, 25-07-01 (J-61-485).

\section{Chara fragilis Desv.}

Se desarrolla tanto en aguas corrientes como estancadas, generalmente dulces o con débil mineralización, estacionales o permanentes. Primera cita provincial.

MÁLAGA. Antequera: Laguna del Viso, 30SUF6997; F. Ortega, 10-07-01 (J-61-486); Laguna de la Caja, 30SUF6998; F. Ortega, 10-07-01 (J-61487); Laguna de las Lomas, 30SUF7198; F. Ortega, 10-07-01 (J-61-488). Archidona: Charca Los Hoyos, 30SUG8505; F. Ortega, 10-07-01 (J-61-489); Laguna Grande, 30SUG8407; F. Ortega, 25-07-01 (J-61-490).

\section{Chara galioides DC.}

Coloniza aguas salinas temporales.

MÁlAGA. Alameda: Laguna de la Ratosa, 30SUG4819; F. Ortega, 27-07-01 (J-61-491). Fuente de Piedra. Cantera del cerro del Palo, 30SUG4510; F. Ortega, 27-07-01 (J-61-492).

Lamprothamnium papulosum (Wallr.) J. Groves.

Típica y exclusiva de aguas saladas, someras y estacionales, tanto en ambientes litorales como continentales.

MÁlAGA. Alameda: Laguna de la Ratosa, 30SUG4819; F. Ortega, 27-07-01 (J-61-493).

\section{Ruppiaceae}

Ruppia drepanensis Tin ex Guss.

Hidrófito halófilo de aguas continentales y temporales, de moderada a elevada salinidad.

MÁLAGA. Alameda: Laguna de la Ratosa, 30SUG4819; F. Ortega, 27-07-01 (J-61-494).

\section{Ruppia maritima L. var. maritima}

Especie de aguas saladas litorales o interiores en ambientes estacionales o permanentes.

MÁLAGA. Humilladero: Laguna de la Serafina Chica, 30SUG4716; F. Ortega, 19-06-01 (J-61-495). Fuente de Piedra: Cantera del cerro del Palo, 30SUG4510; F. Ortega, 27-07-01 (J-61-496).

\section{Zannichelliaceae}

\section{Zannichellia palustris $\mathrm{L}$.}

Macrófito acuático asociado a praderas sumergidas en aguas de moderada salinidad.

MÁlAGA. Humilladero: Laguna de la Serafina Chica, 30SUG4716; F. Ortega, 19-06-01 (J-61-497).

Zannichellia contorta (Desf.) Cham. \& Schlecht.

Planta endémica del mediterráneo occidental que vive en aguas corrientes y limpias, bien oxigenadas y con elevada proporción de calcio.

MÁLAGA. Archidona: Manantial, 30SUG8307; F. Ortega, 25-07-01 (J-61-498).

Althenia orientalis (Tzvelev) García Murillo \& Talavera.

De carácter marcadamente halófilo, coloniza depresiones encharcadas a menudo con salinidades superiores a la del agua marina.

MÁLAGA. Alameda: Laguna de la Ratosa, 30SUG4819 (4919); F. Ortega, 27-07-01 (J-61-499).

\section{Potamogetonaceae}

\section{Potamogeton natans L.}

Hidrófito de aguas remansadas y dulces, de tipo carbonatado.

MÁlAGA. Antequera: Laguna del Viso, 30SUF6997; F. Ortega, 10-07-01 (J-61-500).

\section{Potamogeton pectinatus $\mathrm{L}$.}

Aparece en aguas dulces a moderadamente salinas, tanto estacionales como permanentes. 


\begin{tabular}{lc}
\hline HUMEDALES & CONDUCTIVIDAD $(\mathrm{mS})$ \\
\hline Cantera del Cerro del Palo & 35,00 \\
Laguna de la Serafina Chica & 31,80 \\
Laguna de la Ratosa & 29,70 \\
Laguna Chica & 7,30 \\
Laguna Grande & 3,90 \\
Manantial & 3,12 \\
Laguna del Cortijo Aguilar & 1,00 \\
Charca de los Hoyos & 0,72 \\
Laguna de las Lomas & 0,42 \\
Charca ganadera & 0,28 \\
Laguna de Siete Novias & 0,17 \\
Laguna del Viso & 0,11 \\
Laguna de la Caja & - \\
\hline
\end{tabular}

Tabla 1. Valores de conductividad medidos en los humedales muestreados de la provincia de Málaga. No se disponen de datos sobre la laguna de la Caja. Conductivity values measured in wetlands sampled in Málaga province. Data were not available for La Caja lake.

MÁlAGA. Archidona: Laguna Grande, 30SUG8407; F. Ortega, 25-07-01 (J-61-501); Laguna Chica, 30SUG8306; F. Ortega, 25-07-01 (J61-502).

\section{Polygonaceae}

\section{Polygonum amphibium L.}

Aparece formando parte de comunidades de aguas remansadas, permanentes o semipermanentes, dulces y de tipo carbonatado.

MÁlAGA. Antequera: Laguna de la Caja, 30SUF6998; F. Ortega, 10-07-01 (J-61-503). Aparece junto a Myriophyllum sp.

\section{Haloragaceae}

\section{Myriophyllum spicatum L.}

Origina formaciones densas en lagunas de aguas estacionales o semipermanentes, dulces o subsalinas y con diferentes grados de eutrofia.

MÁlAGA. Archidona: Laguna Chica, 30SUG8306; F. Ortega, 25-07-01 (J-61-505).

La tabla 1 muestra los valores de conductividad medidos en los humedales muestreados.

Los datos obtenidos muestran claramente la necesidad de este tipo de revisiones para el mejor conocimiento de la vegetación acuática de nuestros humedales, sobre todo para el caso de los carófitos, ya que en este territorio los datos sobre este grupo de algas son bastantes escasos. Respecto a las revisiones de sistemas previamente estudiados por otros autores, los datos obtenidos muestran la presencia de tres nuevas especies en dichos ecosistemas. Tal es el caso de la presencia de Chara hispida y Ruppia maritima en la laguna de la Serafina Chica, que había sido previamente estudiada por Asensi y Nieto (1981) y por Nieto et al. (1997). Lamentablemente este humedal ha sido recientemente excavado, lo que junto a la existencia de un canal de drenaje pueden poner en peligro la permanencia de esta interesante vegetación acuática. De igual manera, y en comparación con estos mismos trabajos anteriores y otros (Cirujano et al., 1992; García Murillo et al., 1994), aparece igualmente por primera vez Chara galioides en la laguna de la Ratosa. El resto de referencias en estos humedales, no son nuevas pero confirman la continuidad de estas poblaciones, lo que puede entenderse como una buena gestión de nuestros humedales, sobre todo en aquellos que presentan algún grado de protección legal.

Con relación al estudio de nuevas 
localidades, los datos obtenidos nos han permitido dar citas, algunas de las cuales constituyen la primera referencia para la provincia de Málaga. Digna de reseñar es la presencia de Zannichellia contorta, una especie considerada como vulnerable en la Lista Roja de la Flora Vascular Española (VV.AA., 2000) a consecuencia de su reducida área de distribución y que con esta nueva cita amplia dicho areal.

AGRADECIMIENTOS. Queremos agradecer a la Consejería de Medio Ambiente, Delegación provincial de Málaga, y en especial a D. Manuel Rendón los permisos concedidos para la realización de los muestreos en los espacios protegidos de la provincia de Málaga.

\section{BIBLIOGRAFÍA}

ASENSI, A. y J.M. NIETO-1981- Vegetación acuática, halófila y halonitrófila de la provincia de Málaga. Trabajos y Monografías del Departamento de Botánica de Málaga 2: 105-122

BLINDOW, I. -2000- Distribution of charophytes along the Swedish coast in relation to salinity and eutrophication. Internat. Rev. Hydrobiol. 85: 707-717.

CIRUJANO, S., M. VELAYOS RODRÍGUEZ, F. CASTILLA LATTKE y M. GIL PINILLA 1992- Criterios botánicos para la valoración de las lagunas y humedales españoles (Península Ibérica y las islas Baleares). Ministerio de Agricultura, Pesca y Alimentación. 456 pp.

CIRUJANO, S. -1995- Flora y vegetación de las lagunas y humedales de la provincia de Cuenca. Junta de Comunidades de Castilla-La Mancha, CSIC, Real Jardín Botánico de Madrid. 224 pp.

COMELLES, M. -1985-Clave de identificación de las especies de carófitos de la Península Ibérica. Asociación Española de Limnología. 35 pp.

DANTíN, J. -1940- La aridez y el endorreismo en España. El endorreismo Bético. Estudios Geográficos 1: 75-117.

DOWNING, J.A. \& M.R. ANDERSON -1985Estimating the standing biomass of aquatic macrophytes. Can. J. Fish. Aquat. Sci. 42: 18601869.
GARCÍA MURILLO, P. y S. TALAVERA - 1986 - El género Althenia Petit. Lagascalia 14 (1): 102-114.

GARCÍA MURILLO, P., S. CIRUJANO y F.J. GONZÁLEZ MINERO -1994- Contribución al conocimiento de los carófitos del SO. de la Península Ibérica. Studia Botanica 13: 221-225.

GOSLEE, S.C., R.P. BROOKS \& C.A. COLE 1997- Plants as indicators of wetland water source. Plant Ecology 131: 199-206.

GRILLAS, P., P. GARCÍA MURILLO, O. GEERTZHANSEN, N. MARBÁ, C. MONTES, C.M. DUARTE, L. TAN HAM \& A. CROSSMANN 1993- Submerged macrophyte seed bank in a Mediterranean temporary marsh: abundance and relationship with stablished vegetation. Oecologia 94: 1-6.

LANDERS, D.H. -1982- Effects of naturally senescing aquatic macrophytes on nutrient chemistry and chlorophyll-a of surrounding waters. Limnol. Oceanogr. 27: 428-439.

MARTÍNEZ PARRAS, J.M. -1984- La vegetación lacustre de la depresión de Antequera (Andalucía). Collect. Botanica 15: 289-306.

NIETO, J.M., R.M. CONDE-ÁLVAREZ, M.A. ARREBOLA BAUTISTA y A. FLORES-MOYA -1997- Nuevas citas de macrófitos acuáticos de las zonas húmedas de la provincia de Málaga. Acta Bot. Malacitana 22: 247-248.

TALAVERA, S., P. GARCíA MURILlO y $\mathrm{H}$. SMITH -1986- Sobre el género Zannichellia L. (Zannichelliaceae). Lagascalia 14(2): 241-271.

VALDÉS, B., S. TALAVERA y E. FERNÁNDEZGALIANO (eds.). -1987- Flora vascular de Andalucía Occidental I, II, III. Ed. Kestrell. Barcelona.

VELAYOS, M. -1988- Acotaciones a Ranunculus subgénero Batrachium (DC) A. Gray: tratamiento taxonómico general y estudio de la variabilidad de $\mathrm{R}$. peltatus. Anales Jard. Bot. Madrid 45 (1): 331-332

VV. AA. -2000- Lista roja de la flora vascular española (valoración según categorías UICN). Conservación Vegetal 6 (extra): 11-38.

Aceptado para su publicación en septiembre de 2001

Dirección de los autores. Departamento de Biología Animal, Biología Vegetal y Ecología; Facultad de Ciencias Experimentales. Universidad de Jaén; Paraje de Las Lagunillas s/n; E-23071 Jaén 